\title{
Sperm DNA Integrity and Meiotic Behavior Assessment in an Infertile Male Carrier of a 9qh+++ Polymorphism
}

\author{
A. García-Peiróo, ${ }^{1,2}$ M. Oliver-Bonet, ${ }^{1,3}$ J. Navarro, ${ }^{1}$ C. Abad, ${ }^{4}$ M. Guitart, ${ }^{5}$ \\ M. J. Amengual, ${ }^{5}$ and J. Benet ${ }^{1}$ \\ ${ }^{1}$ Unitat de Biologia Cel-lular i Genètica Mèdica, Facultat de Medicina, Universitat Autònoma de Barcelona, 08193 Bellaterra, Spain \\ ${ }^{2}$ Càtedra de Recerca Eugin-UAB, Universitat Autònoma de Barcelona, 08193 Bellaterra, Spain \\ ${ }^{3}$ Unitat d'Investigació Hospital Universitari Son Dureta, 07014 Palma de Mallorca, Spain \\ ${ }^{4}$ Servei d'Urologia, Consorci Hospitalari Parc Taulí, 08208 Sabadell, Spain \\ ${ }^{5}$ UDIAT, Consorci Hospitalari Parc Tauli, 08208 Sabadell, Spain
}

Correspondence should be addressed to A. García-Peiró, agusti.garcia@uab.es and J. Benet, jordi.benet@uab.cat

Received 5 August 2010; Revised 29 October 2010; Accepted 1 November 2010

Academic Editor: Paul W. Doetsch

Copyright ( $) 2011$ A. García-Peiró et al. This is an open access article distributed under the Creative Commons Attribution License, which permits unrestricted use, distribution, and reproduction in any medium, provided the original work is properly cited.

\begin{abstract}
Although several reports on male infertility suggest a relationship between chromosome 9 polymorphisms and infertility, the effects on the phenotype have not been extensively reported. In this study, an infertile patient was found to carry a 9qh+++ chromosome. The flow cytometric TUNEL assay and SCD test have been applied to characterize sperm DNA integrity. In order to assess its meiotic behaviour, synapsis, recombination, and aneuploidy, analyses have been also performed. Sperm DNA fragmentation (SDF) was $77.81 \%$ and $87 \%$ for the TUNEL and SCD tests, respectively. Ninety-two percent of pachytene cells analyzed showed meiotic abnormalities. The mean number of MLH1 foci per pachytene in the control group was higher (49) than the mean found in the $9 \mathrm{qh}+++$ patient $(38)(P<.0001)$. In spermatozoa, significant increases of disomy rates were observed for chromosome 18 and for the sex chromosomes $(P<.0001)$. These disturbances could be present in other male carriers of a less marked 9qh+.
\end{abstract}

\section{Introduction}

In the last few years, some papers have reported a high incidence of heterochromatin variants in infertile men [1-3]. Paracentric heterochromatin variants usually occur on the long arms of chromosomes 1, 9, 16, and distal heterochromatin of the Y chromosome [1]. In particular, the heterochromatin polymorphism of chromosome 9 is the structural variant most frequently present in infertile men $[2,3]$. In this respect, some authors have suggested that the presence of some heterochromatin polymorphisms may make synapsis difficult, delaying or even preventing it and, as a consequence, may cause the reduction in both sperm number and quality, impairing the fertility of the patient [4].

More recently, several studies have shown that sperm DNA integrity is a highly limiting factor for the correct transmission of paternal genetic information. This could disturb both the fertilization and embryo-development processes $[5,6]$. While recent data about sperm DNA integrity in balanced chromosomes rearranged carriers have been published $[7,8]$, no data from carriers of heterochromatin polymorphism have been published to-date so, consequently, a lack of information about the effects on the phenotype is still present. In order to fill, at least in part, this gap, meiotic, aneuploidy, and sperm DNA integrity analyses have been performed in a carrier of the polymorphic $9 \mathrm{qh}+++$ variant.

\section{Material and Methods}

2.1. Donor and Sample Treatment. The donor is a 36-yearold infertile male with severe oligoasthenoteratozoospermia. 


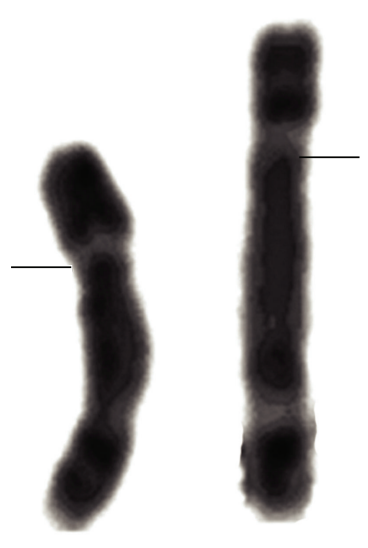

(a)

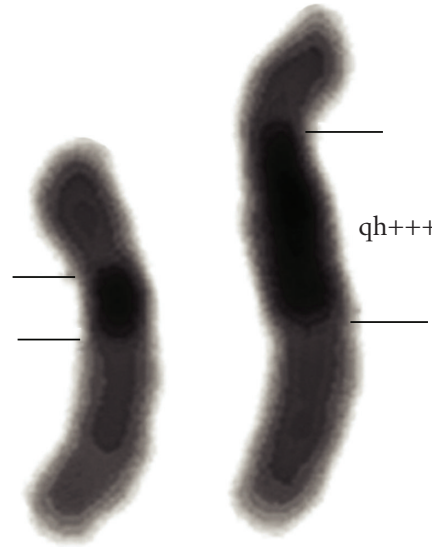

(b)

Figure 1: Karyotype analysis shows a 9qh+++ heterozygous state. (a) G-banding of homologous 9 chromosomes. Abnormal chromosome 9 (right) showing an increased length in the q arm compared to its homologous chromosome (left). Horizontal line indicates centromere position. (b) C-banding shows an increase in the heterochromatin region of chromosome 9. Horizontal lines set the limits of the qh region.

Physical examination showed testes with a slightly diminished volume and consistency. Hormonal assays showed FSH and LH within normal levels. According to World Health Organization recommendations [9], sperm count was estimated to be 2.8 million per $\mathrm{mL}$. No sperm with progressive motility was observed and only $3 \%$ of sperm showed nonprogressive motility. Standard protocols for Gand C-banding analysis were performed on lymphocyte metaphases showing the presence of a $9 \mathrm{qh}+++$ chromosome in which the pericentromeric heterochromatin block tripled the normal length (Figure 1).

For synapsis and recombination analyses, a testicular biopsy was obtained from the patient and five control men, undergoing vasectomy or reversal vasectomy, under a local anaesthesia [10]. To perform DNA integrity tests and the aneuploidy study, a semen sample from the patient and three control men of proven fertility was obtained by masturbation after three days of sexual abstinence. Written consent was given by all patients, and the study was approved by the Institutional Ethics Committee.

Fresh ejaculate was allowed to liquefy, mixed 1:1 with cryopreservation medium (14\% (v/v) glycerol, $30 \%(\mathrm{v} / \mathrm{v})$ egg yolk, $1.98 \%(\mathrm{w} / \mathrm{v})$ glucose, and $1.72 \%(\mathrm{w} / \mathrm{v})$ sodium citrate), aliquoted and incubated overnight at $80^{\circ} \mathrm{C}$ in an isopropanol bath and then plunged directly into liquid nitrogen until the experiment was performed.

For synapsis and recombination analyses, a modification of the drying-down technique [11] was used to obtain meiotic cells from the testicular tissue. Briefly, the tissue was incubated for an hour at room temperature in a hypotonic solution (sodium citrate $1 \%(\mathrm{w} / \mathrm{v})$ ). After incubation, $20 \mu \mathrm{l}$ of $0.1 \mathrm{M}$ sucrose solution were added to the tissue and testicular tubules were shredded using two fine watchmaker forceps until a cell suspension was obtained. This cell suspension was then recovered and spread on a slide previously soaked in $1 \%(\mathrm{w} / \mathrm{v})$ paraformaldehyde. The slide was placed in a humid chamber and allowed to dry overnight. Finally, the slide was washed in 0.04\% (v/v) Photo-Flo (Eastman Kodak SA; Genève, Switzerland) for 4 min at room temperature and air-dried.

2.2. TUNEL Assay. For terminal transferase dUTP nickend labeling (TUNEL), the in situ cell death detection kit, from Roche (Ref. 11684795910, Roche Diagnostic GmbH; Penzberg, Germany), was used as previously described [12]. This assay quantifies, by flow cytometer or fluorescent microscopy, the incorporation of labeled deoxyuridine triphosphate (dUTP) at the sites of DNA breaks in a reaction catalyzed by the enzyme deoxynucleotidyl transferase enzyme. Semen samples from the patient and three fertile and chromosomally normal donors were washed twice in PBS and the concentration was adjusted to $20 \times 10^{6}$ cells $/ \mathrm{mL}$. Two-hundred microliters of this sperm suspension were fixed an equal volume of $4 \%(\mathrm{w} / \mathrm{v})$ paraformaldehyde for 1 hour at room temperature and then washed in PBS supplemented with $1 \%(\mathrm{v} / \mathrm{v})$ bovine serum albumin (BSA; Sigma Chemicals). Sperm cells were permeabilized using $0.1 \%(\mathrm{v} / \mathrm{v})$ Triton $\mathrm{X}-100$ in $0.1 \%(\mathrm{w} / \mathrm{v})$ sodium citrate for 2 minutes in ice and then washed twice in PBS supplemented with $1 \%$ BSA. The pellet was incubated in $50 \mu \mathrm{L}$ of a mix containing $45 \mu \mathrm{L}$ of the label solution plus $5 \mu \mathrm{L}$ of the terminal deoxynucleotidyl transferase (TdT) enzyme for 1 hour at $37^{\circ} \mathrm{C}$ in the dark. The sample was then washed twice using $1 \%$ BSA in PBS. The negative control was incubated without the TdT enzyme and the positive control was prepared before the labeling reaction with an additional treatment with DNAse I (Roche Diagnostic GmbH; Penzberg, Germany), 100 IU, for 10 minutes at $37^{\circ} \mathrm{C}$.

In order to perform flow cytometry analysis, the final pellet from the sperm sample was resuspended in a final volume of $1 \mathrm{~mL}$ PBS. Green fluorescence (TUNEL-positive cells) was measured using a $530 \mathrm{~nm} \pm 30 \mathrm{~nm}$ band-pass filter. A total of 10000 events were measured at a flow rate of 


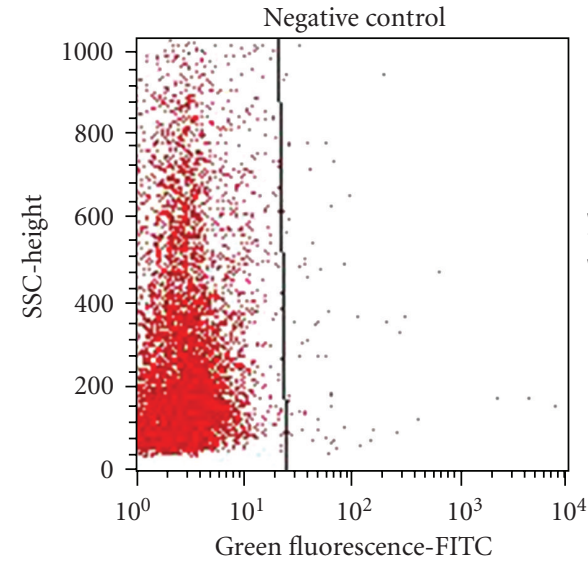

(a)

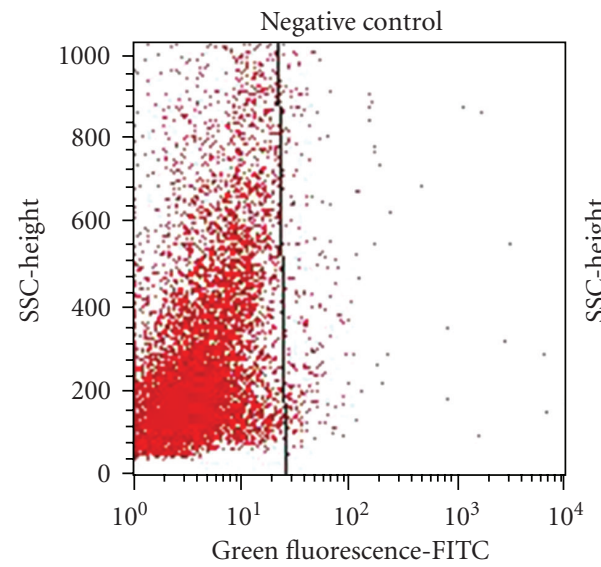

(d)

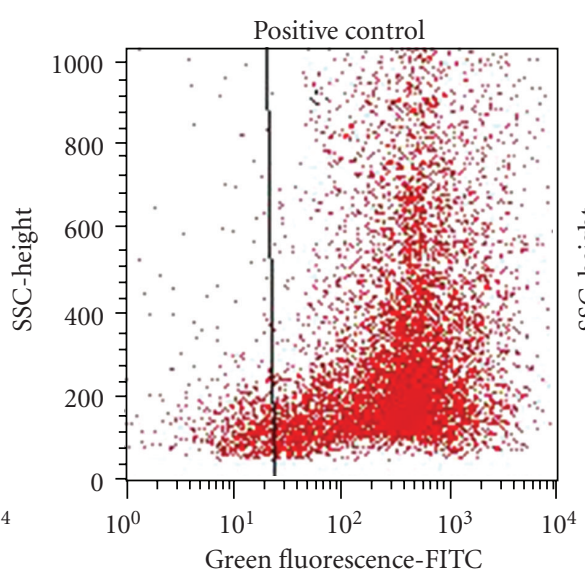

(b)

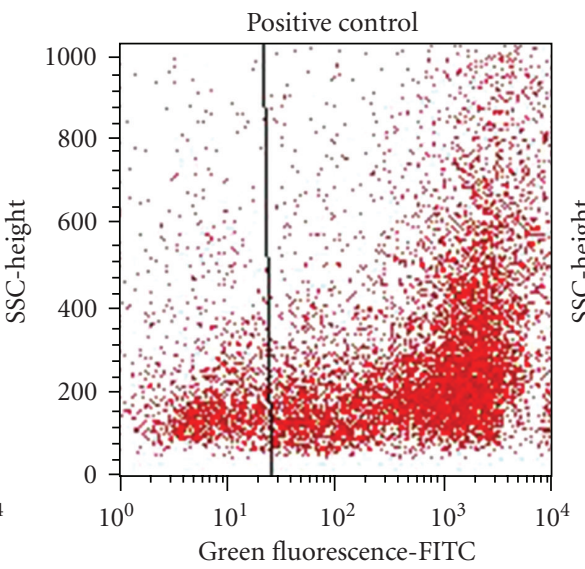

(e)

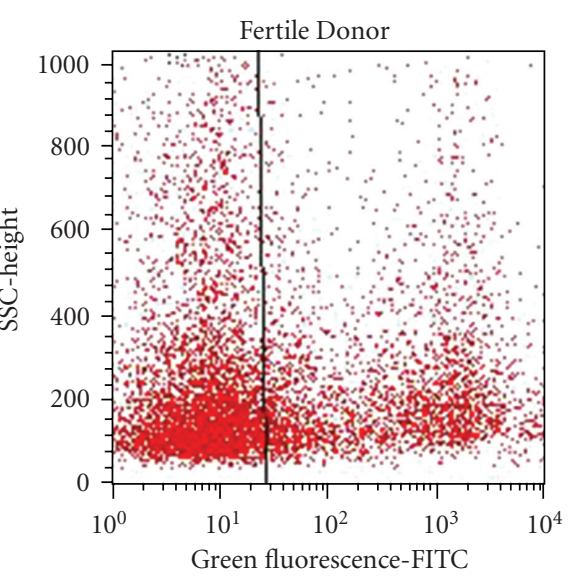

(c)

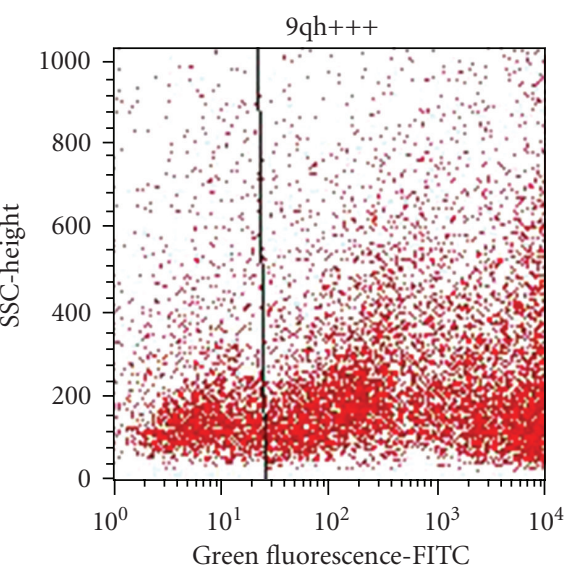

(f)

Figure 2: Cytogram for TUNEL. (a, d) The negative control was incubated without the TdT enzyme. (b, e) Positive control was prepared before the labeling reaction with an additional treatment with DNAse I. (c, f) The TUNEL-positive cell in the fertile donor and patient sample was measured, respectively, with respect to the negative-control sperm population using a $530 \mathrm{~nm} \pm 30 \mathrm{~nm}$ band-pass filter. A total of 10000 events were measured at a flow rate of 200-300 cells/s on a flow cytometer.

200-300 cells/s on a flow cytometer (FACSCalibur; Becton Dickinson, NJ, USA). Data were processed by CELLQUEST analysis software (Becton Dickinson).

2.3. SCD Test. For the Sperm Chromatin Dispersion test (SCDt), the Halosperm kit was used (Chromacell SL; Madrid, Spain). The SCD test is based on the principle that sperm with fragmented DNA fails to produce the characteristic halo of dispersed DNA loops that is observed in nonfragmented DNA sperm [13]. The semen samples from the patient and from three fertile and chromosomally normal donors were washed twice in PBS and the concentration was adjusted to $20 \times 10^{6}$ cells $/ \mathrm{mL}$. Low-melting-point agarose was melted in a water bath at $90^{\circ} \mathrm{C}-100^{\circ} \mathrm{C}$ for $5 \mathrm{~min}$ and placed in water at $37^{\circ} \mathrm{C}$ for $5 \mathrm{~min}$ to allow for equilibration. Then, $60 \mu \mathrm{L}$ of the semen sample were mixed with agarose and $20 \mu \mathrm{L}$ of the semen-agarose mixture were pipetted onto an agarose-coated slide, covered with a coverslip and left at $4^{\circ} \mathrm{C}$ for $5 \mathrm{~min}$. The coverslip was gently removed and immersed in an acid solution for $7 \mathrm{~min}$, washed for $5 \mathrm{~min}$ with distilled water, and incubated in $10 \mathrm{~mL}$ of the lysing solution for $25 \mathrm{~min}$. After washing, the slides were dehydrated in $70 \%$, 90\%, and $100 \%$ ethanol for $2 \mathrm{~min}$ each and then air-dried. Slides were stained for fluorescence microscopy using DAPI ( $2 \mu \mathrm{g} / \mathrm{mL})$ (Roche Diagnostics; Barcelona, Spain) in Vectashield (Vector Laboratories; Burlingame, CA). The positive control was prepared with an additional treatment with DNAse I (Roche Diagnostic GmbH; Penzberg, Germany), $100 \mathrm{IU}$, for 10 minutes at $37^{\circ} \mathrm{C}$. For this study, 300 spermatozoa were scored and the percentage of sperm with fragmented DNA is referred to as sperm DNA fragmentation (SDF).

2.4. Sperm Aneuploidy Study. The sperm aneuploidy study was carried out using Fluorescence In Situ Hybridization (FISH). Frozen samples from three fertile control donors and the patient were thawed in a $37^{\circ} \mathrm{C}$ bath for 30 seconds and then washed in $0.9 \%(\mathrm{w} / \mathrm{v}) \mathrm{NaCl}$ to remove the cryoprotectant, fixed, and decondensed following a protocol previously described [14]. Three-color FISH was 


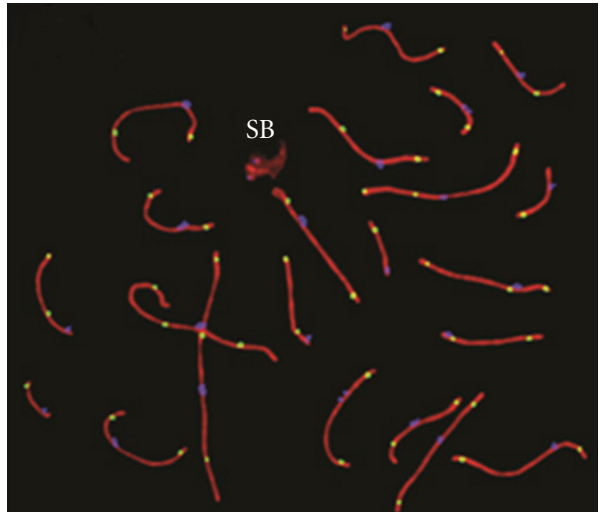

(a)

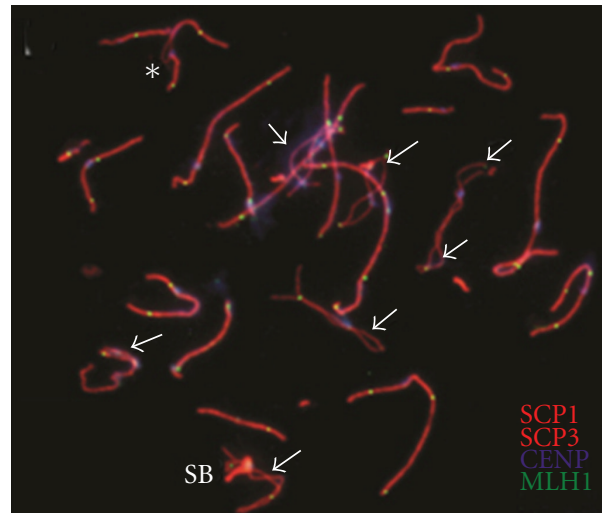

(b)

Figure 3: Immunolabeled pachytene cells with synaptonemal complexes. (a) Representative image from the control group showing the normal morphology of the synaptonemal complex and sex body (SB). (b) Representative image of the cytological analysis of the patient's cells. Arrowheads indicate multiple asynaptic regions in the autosomal synaptonemal complex; an asterisk indicates a loop of asynapsis as a consequence of pericentromeric heterochromatin polymorphism of chromosome 9. SCP1 and SCP3 indicate synaptonemal complexes in red; CENP indicates centromere in blue, and MLH1 (mut L homolog 1) indicates recombination foci in green.

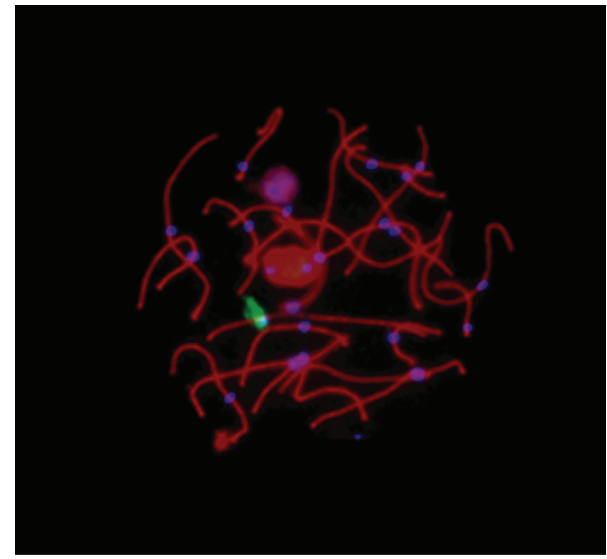

(a)

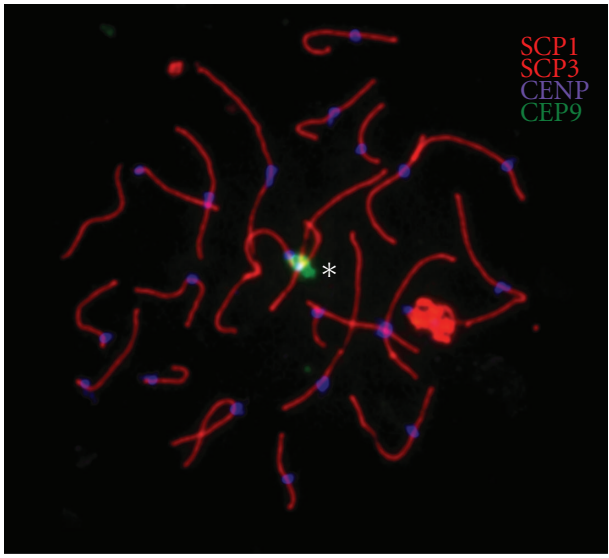

(b)

FIGURE 4: Images show pachytene cells with the bivalent 9 synaptonemal complex identified by FISH analysis. (a) Representative image from a control donor. (b) Representative image from the $9 \mathrm{qh}+++$ patient. An asterisk indicates the loop in the pericentromeric region; SCP1 and SCP3 indicate synaptonemal complexes in red; CENP indicates centromere in blue, and chromosome enumeration probe 9 (CEP9) indicates bivalent 9 in green.

performed with a combination of centromeric probes for chromosomes 18, X, and Y. Following the protocol recommended by the commercial provider (AneuVysion EC DNA Prove Kit; Vysis Inc; Woodcreek, IL, USA), slides were denatured for $5 \mathrm{~min}$ in a $70 \%(\mathrm{v} / \mathrm{v})$ formamide $2 \mathrm{x}$ standard saline citrate $(2 \mathrm{xSSC})$ solution prewarmed at $70 \% \pm 1{ }^{\circ} \mathrm{C}$ in a waterbath, passed through three ethanol series $(70 \%$, $90 \%, 100 \%)$ and air-dried. Five $\mu \mathrm{l}$ of the denatured mixprobe were applied to each slide, and $18 \mathrm{~mm} \times 18 \mathrm{~mm}$ coverslips were added and sealed with rubber cement. Slides were incubated overnight at $37^{\circ} \mathrm{C}$. After incubation, slides were washed following the manufacturer's instructions, dehydrated, and counterstained with antifade (Vector Laboratories, Inc.; Burlingame, CA, USA) containing DAPI at a concentration of $0.032 \mathrm{ng} / \mathrm{mL}$ (Sigma; Madrid, Spain). Hybridization signals were observed using an Olympus Bx60 photomicroscope (Olympus Optical Co.; Hamburg, Germany) with a triple filter for DAPI/FICT/PI. Images were captured and produced by a Cytovision system (Applied Imaging; Sunderland, UK).

From three control donors, a total of 31134 spermatozoa were scored. Moreover, 616 spermatozoa were scored from the $9 \mathrm{qh}+++$ patient. Strict criteria were applied: only individual, well-delineated and intact sperm nuclei were evaluated, and a sperm head was scored as disomic when it displayed two clear signals for the same chromosome, which were of similar size, color and intensity, and separated by at least one fluorescence domain. 
2.5. Synapsis and Recombination Study. Immunocytology of spermatocytes from the control group and the patient sample was performed following Barlow and Hulten [15]. Four primary antibodies were used: rabbit anti-synaptonemal complex protein-3 (SCP3) [16] and rabbit anti-SCP1 [17] (both gifts from Dr. Christa Heyting, University of Wageningen, The Netherlands), anti-centromere protein (CENP) (CREST serum kindly provided by Dr. William Earnshaw), and mouse anti-MLH1 protein (Pharmingen; San Diego, CA, USA). The four primary antibodies were applied at $1: 1000,1: 1000,1: 1000$, and $1: 500$, respectively, in PBT (PBS, $0.15 \%(\mathrm{v} / \mathrm{v})$ Fetal Calf Serum, $0.1 \%$ (v/v) Tween 20) overnight at room temperature. Fluorescence secondary antibodies were applied in two rounds. The first round included a combination of TRITC-conjugated goat antirabbit IgG antibody and FICT-conjugated goat anti-mouse IgG antibody (both from Sigma; Madrid, Spain) at 1:500 in PBT for $2 \mathrm{~h}$ at room temperature. The last round was performed using the Zenon Pacific Blue Rabbit IgG Labeling Kit (Molecular Probes, Spain) to label an unconjugated rabbit anti-human antibody. Incubation time was 40 minutes at room temperature. After three 5-minute washes in PBT and a brief rinse in distilled water, slides were allowed to air-dry. Antifade (Vector lab Inc; Burlingame, CA, USA) was applied to each slide. Evaluation was made using a fluorescent photomicroscope (Olympus Optical Co.; Hamburg, Germany) and all observed pachytene nuclei with antiMLH1 antibody foci were captured and processed using a Power Macintosh G3 with SmartCapture software (Digital Scientific; Cambridge, UK).

2.6. Chromosome 9 FISH. A specific CEP9 SpectrumGreen probe (Vysis, Abbott Molecular Inc; Des Plaines, IL, USA) was used for the bivalent 9 identification. The spectrum green probe was hybridized on previously immunostained preparations. Briefly, slides were washed with $2 \mathrm{xSSC}$ pH 7.0 for two minutes, after which they were dehydrated in an ethanol series $(70 \%, 85 \%$, and $100 \%)$, each one for two minutes, and dried at room temperature. Denaturalization of the sample was performed in $70 \%$ formamide for $3 \mathrm{~min}$ at $73^{\circ} \mathrm{C}$ and probe solution was denatured for $5 \mathrm{~min}$ at $73^{\circ} \mathrm{C}$. Hybridization was performed in a humid lightproof container at $37^{\circ} \mathrm{C}$ overnight. After hybridization, the slides were washed in $0.4 \mathrm{xSSC} / 0.3 \% \mathrm{NP} 40$ at $74^{\circ} \mathrm{C}$ for two minutes followed by a second wash with 2 xSSC/0.01\% NP40 at room temperature for 30 seconds. Finally, DNA was counterstained by applying antifade solution containing $125 \mathrm{ng} / \mu \mathrm{l}$ of DAPI. Identification was performed using a fluorescent photomicroscope (Olympus Optical Co.; Hamburg, Germany) equipped with the ISIS digital FISH-imaging system (MetaSystems; Altussheim, Germany).

2.7. Statistical Analysis. The Chi-Square test and Fisher test were applied when needed for qualitative data analysis to determine whether there were significant differences between two groups for the MLH1, aneuploidy percentages of chromosomes X, Y, and 18 and sperm DNA fragmentation variables. The Student's $t$-test and U-Mann-Whitney test
TABle 1: Percentages of sperm with DNA fragmentation in semen of a heterochromatin polymorphism carrier and control group of fertile donors.

\begin{tabular}{lccc}
\hline & Patient & Control $(n=3)$ & $P$ value \\
\hline TUNEL $^{\mathrm{a}}$ & $77.81 \%$ & $15.6 \%$ & $P<.0001$ \\
SCD $^{\mathrm{b}}$ & $87 \%$ & $10.33 \%$ & $P<.0001$ \\
\hline
\end{tabular}

$\overline{\mathrm{a}, \mathrm{b}}$ For the 9qh+++ carrier, 10000 and 300 spermatozoa were analyzed for TUNEL and SCD, respectively. For controls, 30000 and 900 sperm cells were analyzed.

TABLE 2: Values obtained for asynapsis in pachytene cells in the heterochromatin polymorphism carrier.

\begin{tabular}{lcccc}
\hline \multicolumn{3}{c}{ Normal } & \multicolumn{3}{c}{ Anomalous } \\
\hline Synapsis $^{\mathrm{a}}$ & $8 \%$ & \multicolumn{3}{c}{$92 \%$} \\
\hline & Only in & Chromosome & Only in \\
& & chromosome 9 & 9 and others & others \\
& $40 \%$ & $32 \%$ & $20 \%$ \\
\hline
\end{tabular}

${ }^{\mathrm{a}}$ The presence of synaptonemal complex abnormalities was analyzed in 50 pachytene cells.

were applied to quantitative data comparisons. A value of $P<.05$ was considered significant.

\section{Results}

3.1. Sperm DNA Damage Analysis. DNA fragmentation was high regardless of the method applied: $77.81 \%$ and $87 \%$ SDF for the flow cytometric TUNEL assay (Figure 2) and the SCD test, respectively. In the control group $(n=3)$, a mean of $15.6 \%$ and $10.53 \%$ was found for the TUNEL and the SCD tests, respectively. Statistical differences were found $(P<.0001)$ between the SDF values of this patient and those of the control group (Table 1).

3.2. Synapsis Analysis. Figure 3 shows the immunocytogenetic analysis. The presence of asynapsis, SC fragmentation and XY association was evaluated for all autosomal SCs in 50,224 , and 213 pachytene nuclei in the $9 \mathrm{qh}+++$ carrier and in two control groups $[4,10]$, respectively. Table 2 shows the frequencies of synapsis disturbances observed in pachytene cells of the $9 \mathrm{qh}+++$ carrier. We found that $92 \%$ of the analyzed spermatocytes presented some of these abnormalities. Of them, $40 \%$ of the cells presented asynapsis only in chromosome 9 , whereas $32 \%$ presented asynapsis in chromosome 9, plus SC fragmentation, asynapsis in other chromosomes, or XY association with autosomes. Finally, $20 \%$ of the cells presented complete synapsis of chromosome 9 , but there were different alterations affecting other SCs. In the control group, a total of $11.7 \%$ of unsynapsed bivalent regions (splits) were seen. Observed ranges were from 2.4\% to $29.2 \%$ [10]. Differences regarding asynapsis frequency for bivalent 9 were found between the patient and the control group [4] (Figure 4 and Table 3).

3.3. MLH1 Foci Analysis. For recombination analysis, a total of 43 pachytene nuclei were studied and the number of 
TABLE 3: Number and percentage of asynapsis and heterosynapsis found according to the different pachytene stages analyzed for bivalent 9.

\begin{tabular}{|c|c|c|c|c|c|c|}
\hline \multirow[t]{3}{*}{ Stage } & \multirow{2}{*}{\multicolumn{2}{|c|}{$\begin{array}{c}\text { Control }^{\mathrm{a}}(n=213) \\
\text { Asynapsis in } 9 \mathrm{q}\end{array}$}} & \multicolumn{4}{|c|}{ Patient $9 \mathrm{qh}+++(n=50)$} \\
\hline & & & \multicolumn{2}{|c|}{ Asynapsis in $9 q$} & \multicolumn{2}{|c|}{ Heterosynapsis in $9 q$} \\
\hline & $n=43$ & $\%$ & $n=36$ & $\%$ & $n=14$ & $\%$ \\
\hline Early & 30 & 14.1 & 11 & 22 & 3 & 6 \\
\hline Late & 13 & 6.1 & 20 & 40 & 7 & 14 \\
\hline Unknown & - & - & 5 & 10 & 4 & 8 \\
\hline Total (\%) & & 20.2 & & 72 & & 28 \\
\hline
\end{tabular}

${ }^{a}$ Codina-Pascual el al., [4]

MLH1 foci was scored per cell. A significant reduction in the MLH1 foci number has been found in the $9 \mathrm{qh}+++$, when compared with our controls $(P<.0001)$. The mean number of MLH1 foci per cell observed in controls was $48.8 \pm 2.3$, and ranged from 36 to 63 foci per cell, whereas a mean of $38 \pm 8.28$ foci per cell was found in the $9 \mathrm{qh}+++$, ranging from 12 to 50 foci per cell.

3.4. Aneuploidy Assay. A total of 616 sperm from the $9 \mathrm{qh}+++$ carrier and 31,134 sperm from the control group ( $n=3$ ) were analyzed by triple-color FISH. Significant differences were found between these two groups for sex disomies, autosomal disomies of chromosome 18, and diploidy $(P<.0001)$. Results are summarized in Table 4 .

\section{Discussion}

In the present report, a less common variant of chromosome 9 polymorphism has been analyzed, and new evidence that correlates with infertility is provided. The karyotype of the patient showed an enlarged pericentromeric heterochromatin block which triples its normal length, with regards to its homologous chromosome (Figure 1). Significant meiotic alterations, anomalous aneuploidy rates, high-sperm DNA fragmentation, and altered seminogram parameters have been found.

In the clinical examination, the patient showed an impaired fertility status but no other pathologies. Therefore, the occurrence of a polymorphism of such significance seems to have a strong effect on the germ cells, but not on somatic cells. It seems that asynapsis is a determining aspect for the origin of germ-cell collapse $[18,19]$. Thus, the presence of a threshold amount of asynapsed regions could be a sign of abnormal meiotic progression and could trigger apoptosis [4]. In this patient, synaptic disturbances in $92 \%$ of the analyzed pachytene cells were found. Similar results were reported by Solari et al. [20] in their previous work, where all early pachytene cells presented loops of asynapsis in chromosome 9, which disappeared at late-pachytene, probably because of synaptic adjustment. In our case, a high percentage of late-pachytene stage cells (40\%) presented important loops of asynapsis at the pericentromeric region of chromosome 9 (Figure 4(b) and Table 3) probably because the heterochromatin polymorphism is extremely large and the heterologous pairing could not compensate the impairment in time and extension.
TABLe 4: Aneuploidy percentages of chromosomes X, Y, and 18 in the heterochromatin polymorphism carrier and in a chromosomally normal control group.

\begin{tabular}{lccc}
\hline & Patient $^{\mathrm{a}}$ & Control $^{\mathrm{b}}$ & $P$ value \\
\hline Sex disomies & & & \\
$\mathrm{X}, \mathrm{X}$ & $1.3 \%$ & $0.04-0.09 \%$ & $<0.0001^{* *}$ \\
$\mathrm{Y}, \mathrm{Y}$ & $1.1 \%$ & $0.06-0.14 \%$ & $<0.0001^{* *}$ \\
$\mathrm{X}, \mathrm{Y}$ & $1.4 \%$ & $0.14-0.32 \%$ & $<0.0001^{* *}$ \\
\hline Autosomal disomies & & & \\
18,18 & $0.9 \%$ & $0.09-0.17 \%$ & $<0.0001^{* *}$ \\
Diploidy & $2.6 \%$ & $0.15-0.31 \%$ & $<0.0001^{* *}$ \\
\hline
\end{tabular}

a,b 616 and 31134 spermatozoa were analyzed, respectively.

We have also analyzed the frequencies of MLH1 foci and sperm aneuploidy for standard chromosomes $X, Y$, and 18 in the patient and in chromosomally normal controls. In the patient, a significantly low frequency of MLH1 and an increase of aneuploidy for all chromosomes analyzed were found (Table 4). It is generally accepted that abnormal meiotic recombination is associated with aneuploid sperm production because crossing-over is necessary for proper chromosome segregation [21]. A recent report has shown a correlation between meiotic recombination and testicular sperm aneuploidy in the same individual [22].

Concerning DNA integrity, a significant increase in the number of sperm with DNA fragmentation was seen in the patient in comparison with fertile donors (Table 1 and Figure 2). Furthermore, in other infertile patients with different pathologies of the reproductive system, such as varicocele, and even in patients with chromosomal rearrangements, the values of SDF were statistically lower than those observed in this particular case [8]. The principally known causes of DNA fragmentation are apoptosis during the process of spermatogenesis; DNA strand breaks produced during the remodeling of sperm chromatin in spermiogenesis, and DNA fragmentation induced by oxygen radicals $[23,24]$. In this case, apoptosis could be the main cause of DNA damage at the testicular level, thus explaining, the low count of sperm cells found in the seminogram. However, other causes could be responsible for sperm DNA damage. The remodeling of sperm chromatin, the histone-protamine transition, is an exclusive spermiogenetic cellular process that occurs during the round-spermatid to long-spermatid stage. As the integrity of DNA and chromatin depends, in part, on the accurate progression of this process, alterations at this 
level can affect the normal nuclear architecture and induce vulnerability to oxidative stress and to DNA fragmentation $[25,26]$. A few studies have been reported about SDF in carriers of a chromosomal abnormality [7,27] and abnormal SDF values have been found in these patients. Perhaps the biological process may be similar because chromosomal reorganization could interfere with the normal nuclear architecture.

The implication of all of these data could be useful for clinical practice. In particular, it would be interesting to know to what extent which of the disturbances described here, albeit less marked, could be present in other men with heterochromatin polymorphism. The correlation seen between sperm DNA integrity and the presence of heterochromatic polymorphism, if confirmed, can be of help, in order to establish a predictive fertility status, simply by an SDF analysis in this type of carrier.

Further studies focusing on the role of chromosome- 9 heterochromatin during male gametogenesis will be needed in order to elucidate the mechanisms underlying meiotic failure observed in carriers of chromosome-9 polymorphic variants.

\section{Acknowledgments}

The authors wish to thank Raquel Torres for technical assistance and Chuck Simmons for the English revision of this manuscript. This work was supported by the Fondo Investigación Sanitaria (Grant Numbers PI051834, PI080623 to J. Benet), and by the Generalitat de Catalunya (Grant Number 2009 SGR 1107 to J. Benet). A. García-Peiró has a grant from the Càtedra de Recerca Eugin-UAB.

\section{References}

[1] P. F. Madon, A. S. Athalye, and F. R. Parikh, "Polymorphic variants on chromosomes probably play a significant role in infertility," Reproductive BioMedicine Online, vol. 11, no. 6, pp. 726-732, 2005.

[2] K. Yakin, B. Balaban, and B. Urman, "Is there a possible correlation between chromosomal variants and spermatogenesis?" International Journal of Urology, vol. 12, no. 11, pp. 984-989, 2005.

[3] J. Lissitsina, R. Mikelsaar, and M. Punab, "Cytogenetic analyses in infertile men," Archives of Andrology, vol. 52, no. 2, pp. 91-95, 2006.

[4] M. Codina-Pascual, J. Navarro, M. Oliver-Bonet et al., "Behaviour of human heterochromatic regions during the synapsis of homologous chromosomes," Human Reproduction, vol. 21, no. 6, pp. 1490-1497, 2006.

[5] M. Benchaib, V. Braun, J. Lornage et al., "Sperm DNA fragmentation decreases the pregnancy rate in an assisted reproductive technique," Human Reproduction, vol. 18, no. 5, pp. 1023-1028, 2003.

[6] M. Bungum, P. Humaidan, A. Axmon et al., "Sperm DNA integrity assessment in prediction of assisted reproduction technology outcome," Human Reproduction, vol. 22, no. 1, pp. 174-179, 2007.

[7] A. Perrin, E. Caer, M. Oliver-Bonet et al., "DNA fragmentation and meiotic segregation in sperm of carriers of a chromosomal structural abnormality," Fertility and Sterility, vol. 92, no. 2, pp. 583-589, 2009.

[8] A. García-Peiró, J. Martínez-Heredia, M. Oliver-Bonet et al., "Protamine 1 to protamine 2 ratio correlates with dynamic aspects of DNA fragmentation in human sperm," Fertility and Sterility. In press.

[9] World Health Organization, WHO Laboratory Manual for the Examination of Human Semen and Semen-Cervical Mucus Interaction, Cambridge University Press, Cambridge, UK, 4th edition, 1999.

[10] M. Codina-Pascual, M. Oliver-Bonet, J. Navarro et al., "Synapsis and meiotic recombination analyses: MLH1 focus in the XY pair as an indicator," Human Reproduction, vol. 20, no. 8, pp. 2133-2139, 2005.

[11] A. H. F. M. Peters, A. W. Plug, M. J. Van Vugt, and P. De Boer, "A drying-down technique for the spreading of mammalian melocytes from the male and female germline," Chromosome Research, vol. 5, no. 1, pp. 66-68, 1997.

[12] D. Domínguez-Fandos, M. I. Camejo, J. L. Ballescà, and R. Oliva, "Human sperm DNA fragmentation: correlation of TUNEL results as assessed by flow cytometry and optical microscopy," Cytometry A, vol. 71, no. 12, pp. 1011-1018, 2007.

[13] J. L. Fernández, L. Muriel, V. Goyanes et al., "Halosperm is an easy, available, and cost-effective alternative for determining sperm DNA fragmentation," Fertility and Sterility, vol. 84, no. 4, p. 860, 2005.

[14] F. Vidal, M. Moragas, V. Catala et al., "Sephadex filtration and human serum albumin gradients do not select spermatozoa by sex chromosome: a fluorescent in-situ hybridization study," Human Reproduction, vol. 8, no. 10, pp. 1740-1743, 1993.

[15] A. L. Barlow and M. A. Hultén, "Crossing over analysis at pachytene in man," European Journal of Human Genetics, vol. 6, no. 4, pp. 350-358, 1998.

[16] J. H. M. Lammers, H. H. Offenberg, M. Van Aalderen, A. C. G. Vink, A. J. J. Dietrich, and C. Heyting, "The gene encoding a major component of the lateral elements of synaptonemal complexes of the rat is related to X-linked lymphocyteregulated genes," Molecular and Cellular Biology, vol. 14, no. 2, pp. 1137-1146, 1994.

[17] R. L. J. Meuwissen, H. H. Offenberg, A. J. J. Dietrich, A. Riesewijk, M. Van Iersel, and C. Heyting, "A coiled-coil related protein specific for synapsed regions of meiotic prophase chromosomes," EMBO Journal, vol. 11, no. 13, pp. 5091-5100, 1992.

[18] J. Navarro, C. Templado, J. Benet, R. Lange, O. Rajmil, and J. Egozcu e, "Sperm chromosome studies in an infertile man with partial, complete asynapsis of meiotic bivalents," Human Reproduction, vol. 5, no. 2, pp. 227-229, 1990.

[19] F. Sun, P. Turek, C. Greene, E. Ko, A. Rademaker, and R. H. Martin, "Abnormal progression through meiosis in men with nonobstructive azoospermia," Fertility and Sterility, vol. 87, no. 3, pp. 565-571, 2007.

[20] A. J. Solari, R. Ponzio, and G. Rey Valzacchi, "Synaptonemal complex karyotyping in an oligospermic patient with heterochromatin duplication in chromosome n. 9," Medicina, vol. 51, no. 3, pp. 217-221, 1991.

[21] R. H. Martin, "Meiotic errors in human oogenesis and spermatogenesis," Reproductive BioMedicine Online, vol. 16, no. 4, pp. 523-531, 2008.

[22] F. Sun, M. Mikhaail-Philips, M. Oliver-Bonet et al., "Reduced meiotic recombination on the $\mathrm{XY}$ bivalent is correlated with an increased incidence of sex chromosome aneuploidy 
in men with non-obstructive azoospermia," Molecular Human Reproduction, vol. 14, no. 7, pp. 399-404, 2008.

[23] D. Sakkas, E. Seli, D. Bizzaro, N. Tarozzi, and G. C. Manicardi, "Abnormal spermatozoa in the ejaculated: abortive apoptosis and faulty nuclear remodelling during spermatogenesis," Reproductive BioMedicine Online, vol. 7, no. 4, pp. 428-432, 2003.

[24] G. N. De Iuliis, L. K. Thomson, L. A. Mitchell et al., "DNA damage in human spermatozoa is highly correlated with the efficiency of chromatin remodeling and the formation of 8-hydroxy-2'-deoxyguanosine, a marker of oxidative stress," Biology of Reproduction, vol. 81, no. 3, pp. 517-524, 2009.

[25] R. J. Aitken and G. N. De Iuliis, "Origins and consequences of DNA damage in male germ cells," Reproductive BioMedicine Online, vol. 14, no. 6, pp. 727-733, 2007.

[26] N. Torregrosa, D. Domínguez-Fandos, M. I. Camejo et al., "Protamine 2 precursors, protamine 1/protamine 2 ratio, DNA integrity and other sperm parameters in infertile patients," Human Reproduction, vol. 21, no. 8, pp. 2084-2089, 2006.

[27] F. Brugnon, E. Van Assche, G. Verheyen et al., "Study of two markers of apoptosis and meiotic segregation in ejaculated sperm of chromosomal translocation carrier patients," Human Reproduction, vol. 21, no. 3, pp. 685-693, 2006. 

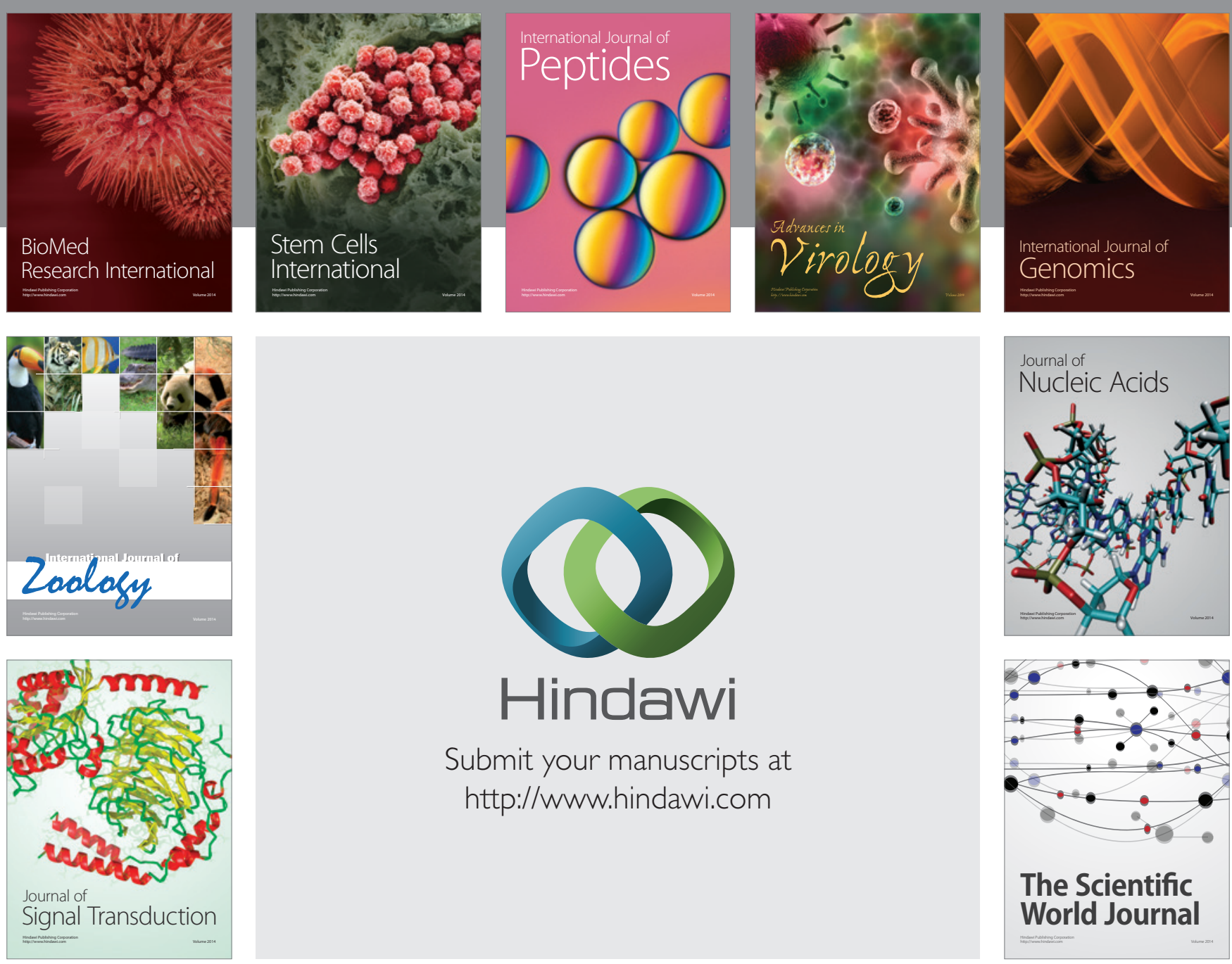

Submit your manuscripts at

http://www.hindawi.com
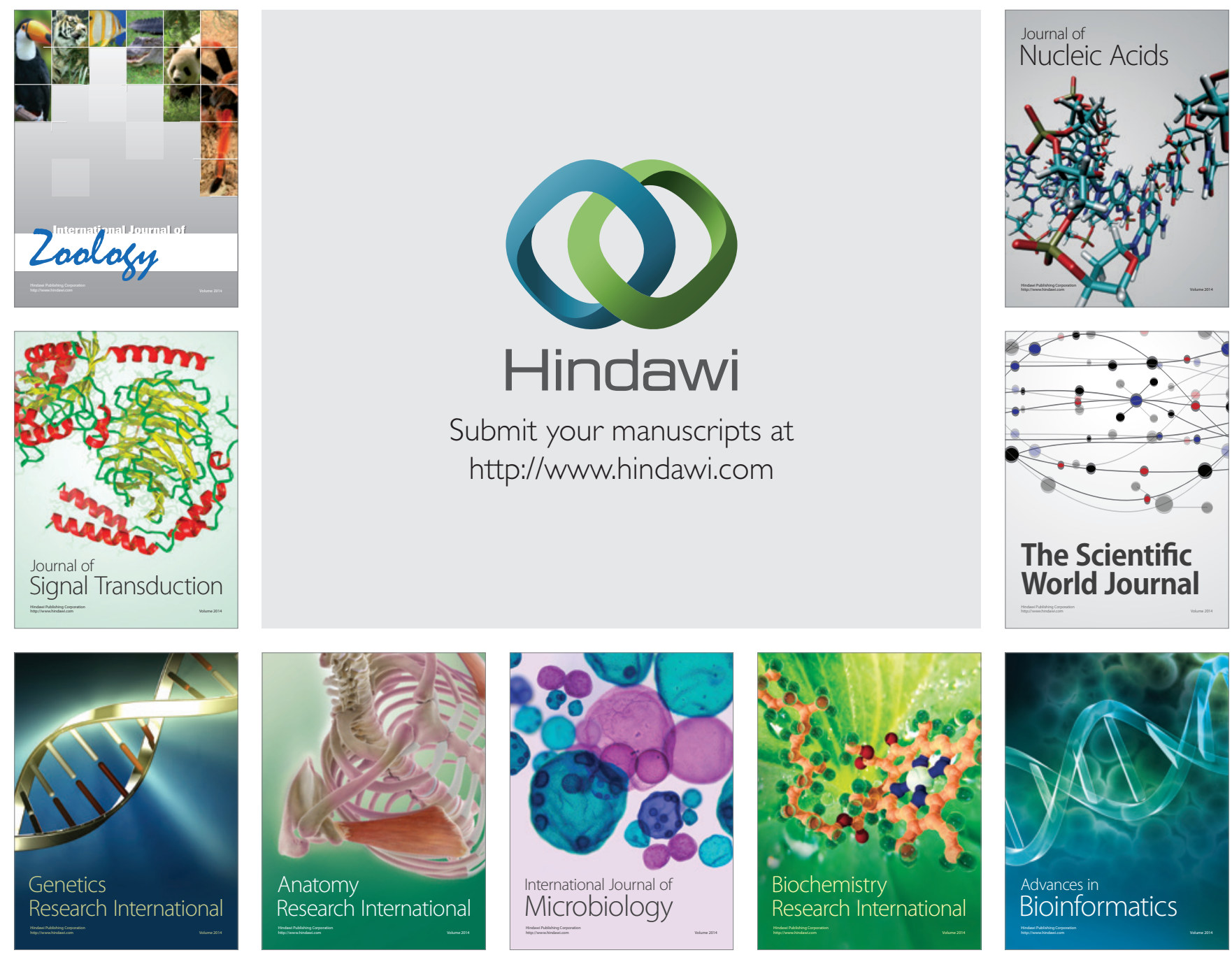

The Scientific World Journal
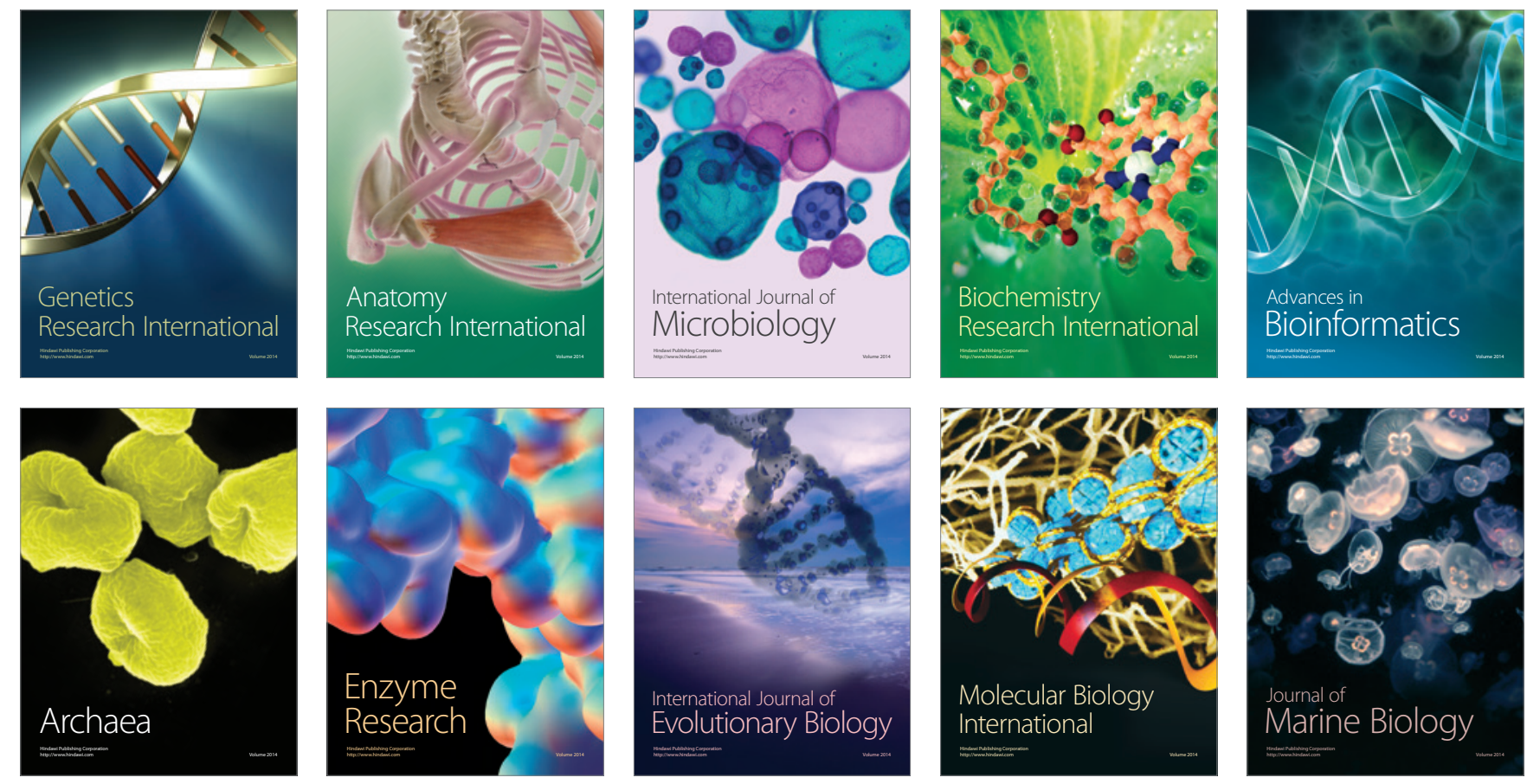